\title{
Noncoplanar volumetric modulated arc therapy for patient with optic nerve sheath meningioma: a case report and literature review
}

\author{
Chi-Yeh Wu ${ }^{1}$, Tzu-Lun Huang, ${ }^{2,3}$, Hsin-Hua Nien ${ }^{4,5}$, Pei-Wei Shueng, ${ }^{1,6}$, Hui-Ju Tien ${ }^{6,7}$, Hsin-Pei Yeh ${ }^{6}$, \\ Chen-Hsi Hsieh ${ }^{1,6,8}$
}

${ }^{1}$ Faculty of Medicine, School of Medicine, National Yang-Ming University, Taipei, Taiwan; ${ }^{2}$ Department of Ophthalmology, Far Eastern Memorial Hospital, New Taipei City, Taiwan; ${ }^{3}$ Department of Electrical Engineering, Yuan Ze University, Chung-Li, Taoyuan, Taiwan; ${ }^{4}$ Department of Radiation Oncology, Cathay General Hospital, Taipei, Taiwan; ${ }^{5}$ School of Medicine, College of Medicine, Fu Jen Catholic University, New Taipei City, Taiwan; ${ }^{6}$ Division of Radiation Oncology, Department of Radiology, Far Eastern Memorial Hospital, New Taipei City, Taiwan; ${ }^{7}$ Department of Biomedical Imaging and Radiological Science, National Yang-Ming University, Taipei, Taiwan; ${ }^{8}$ Institute of Traditional Medicine, School of Medicine, National Yang-Ming University, Taipei, Taiwan

Correspondence to: Chen-Hsi Hsieh, MD, PhD. Division of Radiation Oncology, Department of Radiology, Far Eastern Memorial Hospital, No. 21, Sec. 2, Nanya S. Rd., Banciao Dist., New Taipei City 220, Taiwan. Email: chenciab@gmail.com; chenci28@ym.edu.tw.

\begin{abstract}
A 74-year-old female presented with progressive oculus dexter (OD) visual deterioration caused by optic nerve sheath meningioma (ONSM). The Farnsworth D-15 Dichotomous Color Blindness Test progressed from $3 / 15$ to $1 / 15$ in OD. The visual acuity with correction (VACC) changed from 0.4 to 0.3 in OD after 1 month of medication therapy. A total dose of 54 Gy in 30 fractions was delivered to the ONSM with the noncoplanar volumetric modulated arc therapy (VMAT) technique (Versa HD, Elekta, Crawley, West Sussex, UK). Color discrimination was improved from 1/15 to $13 / 15$ after the patient received 27 Gy in 15 fractions. The VACC of the right eye treated with 54 Gy was improved from 0.3 to 0.6 at 9 months and to 1.0 at 15 months after radiotherapy (RT). There was no neuropathy, visual impairment, cognitive problem or retinopathy upon ophthalmologic examination during or after RT. The size of the tumor was stable, which was confirmed by radiographic studies. The fractionated noncoplanar VMAT technique provides delicate balance between dose coverage and normal tissues protection with well tumor control and improvement in visual function without treatment-induced morbidity.
\end{abstract}

Keywords: Optic nerve sheath meningioma (ONSM); volumetric modulated arc therapy (VMAT); image-guided system; orbital neoplasms; case report

Received: 27 July 2020; Accepted: 11 September 2020; Published: 30 September 2020.

doi: 10.21037/tro-2019-pmc-07

View this article at: http://dx.doi.org/10.21037/tro-2019-pmc-07

\section{Introduction}

Optic nerve sheath meningioma (ONSM) is a rare disease, accounting for $1 \%$ to $2 \%$ of all meningiomas; approximately $92 \%$ of ONSMs are intraorbital in origin, and $95 \%$ of these are unilateral (1). ONSM occurs primarily in females, with a mean age at presentation of 40 years $(1,2)$. The optic pathway compression, proptosis, or ocular motility disorder caused by ONSM progresses slowly (2,3). Ophthalmological findings, together with magnetic resonance imaging (MRI), usually allow establishing the diagnosis of ONSM without biopsy $(2,3)$.

The management of ONSM remains a particular challenge. The National Comprehensive Cancer Network (NCCN) guideline version 1.2019 of central nervous system (CNS) cancers (https://www.ncen.org/professionals/ physician_gls/pdf/cns.pdf) indicates that observation is preferred for asymptomatic small meningiomas. Unfortunately, observation has historically led to poor 
outcomes; for example, $86 \%$ of ONSM patients under observation experienced visual deterioration (1). In patients with one or more tumor- and/or treatmentrelated risk factors, such as proximity to the optic nerve, active treatment by surgery and/or radiotherapy (RT) is recommended by NCCN guidelines. However, in Dutton's reviewed report, there was a significantly increasing risk of vascular injuries through surgery, leading to $94 \%$ of patients with worsened vision and $78 \%$ with loss of light perception, and only $5 \%$ of patients demonstrated an improvement in vision (1). Resection of the tumor is associated with a high risk of blindness (4) and a high rate of local recurrence (5).

Highly conformal fractionated RT [such as threedimensional conformal radiation therapy (3DCRT), intensity-modulated radiation therapy (IMRT), volumetric modulated arc therapy (VMAT) and proton therapy] with 45-54 Gy is also suggested by the NCCN guidelines for grade I meningioma. Recent advances in RT techniques, such as fractionated stereotactic radiotherapy (FSRT) based on three-dimensional treatment planning (6) or IMRT (7), have been recommended for patients unsuitable for surgery and the long-term control rate was $86-100 \%(1,8,9)$. The VMAT technique could allow for IMRT delivery during gantry rotation with dynamic multi-leaf collimator motion, variable dose rates and gantry speed modulation. These characteristics significantly reduces the time and monitor units required to deliver a patient's treatment (10). However, little information is available on the impact of noncoplanar VMAT on ONSM. Herein, we present a first report of primary ONSM patient treated by a fractionated noncoplanar VMAT technique with an encouraging response including long-term efficacy and toxicity outcome. We present the following case in accordance with the CARE reporting checklist (available at http://dx.doi.org/10.21037/ tro-2019-pmc-07).

\section{Case presentation}

\section{Patient history}

A 74-year-old female with a history of hypertension and colon tubule-villous adenoma had suffered from progressive oculus dexter (OD) visual deterioration for 2 or more months without trauma or operation history. She visited the local medical department for visual deterioration, but the cause was undetermined. Therefore, she was referred to the Department of Ophthalmology, Far Eastern Memorial Hospital, Taiwan. Ophthalmological examinations showed visual acuity with correction $\left(\mathrm{VA}_{\mathrm{CC}}\right): 0.4 \mathrm{OD}, 0.6$ over oculus sinister (OS); intraocular pressure: $18 \mathrm{mmHg}$ OD, 17 mmHg OS; Farnsworth D-15 Dichotomous Color Blindness Test: 3/15 OD, 15/15 OS; obvious visual field decreased OD assessed by automated perimetry (Figure 1). The 24-2 Visual Field Test revealed generalized visual field loss over the right eye and arcuate scotoma over the left eye. The mean deviation (MD) was $-27.69 \mathrm{~dB}$ OD and $-11.73 \mathrm{~dB}$ OS. A relative afferent pupillary defect (RAPD) was noted in the right eye with intact extraocular motion and without ptosis. MRI showed a $12 \times 6 \mathrm{~mm}$ eccentric enhanced mass located on the right distal optic nerve, and right ONSM was diagnosed (Figure 2). Prednisolone (1\# po q.i.d.) was used to release the nerve decompression; however, visual impairment with color perception declined progressively ( $\mathrm{VA}_{\mathrm{CC}}$ and color vision in $\mathrm{OD}$ were 0.4 to 0.3 and $3 / 15$ to $1 / 15$, respectively). Thereafter, the patient was transferred to the department of Radiation Oncology.

The patient was treated with Versa HD $^{\mathrm{TM}}$ (Elekta, Crawley, West Sussex, UK) to 54 Gy in 30 fractions within 6 weeks with a VMAT technique. The contouring system was operated using the Pinnacle 3 Treatment Planning System (Philips Healthcare, Madison, WI, USA). The clinical target volumes (CTVs) were defined as the area encompassing the gross tumor. The CTV areas plus $3 \mathrm{~mm}$ were used to generate the planning target volume (PTV). The RT plan with different techniques for the patient were compared (Figure 3). The dose constraints for organs at risk (OAR) were as follows: (I) optic chiasm and optic nerve: maximum dose, 55 Gy; (II) lenses < 10 Gy; and lacrimal gland <35 Gy; (III) one parotid gland was spared to a mean dose of less than $20 \mathrm{~Gy}$ or, if both glands are spared, to less than 25 Gy (mean dose); (IV) brainstem: maximum dose, 54 Gy; (V) spinal cord: maximum dose, 50 Gy.

\section{Plan evaluation}

To compare VMAT and conventional radiation therapy (2DRT), 3DRT and IMRT, the Paddick conformity index (PCI) and uniformity index (UI) were applied. The PCI was originally proposed by Paddick (11) to evaluate the tightness of fit of the PTV to the prescription isodose volume in the treatment plans and was calculated as follows: $\mathrm{PCI}=\left(\mathrm{TV}_{\mathrm{PIV}}{ }^{2}\right)$ / $(\mathrm{TV} \times \mathrm{PIV})$, in which TV is the PTV volume, PIV is the treated volume enclosed by the prescription isodose surface, and $\mathrm{TV}_{\mathrm{PIV}}$ is the volume of the PTV within the prescribed isodose. A PCI value close to unity means more conformity of the dose distribution to the target volume. The UI is 

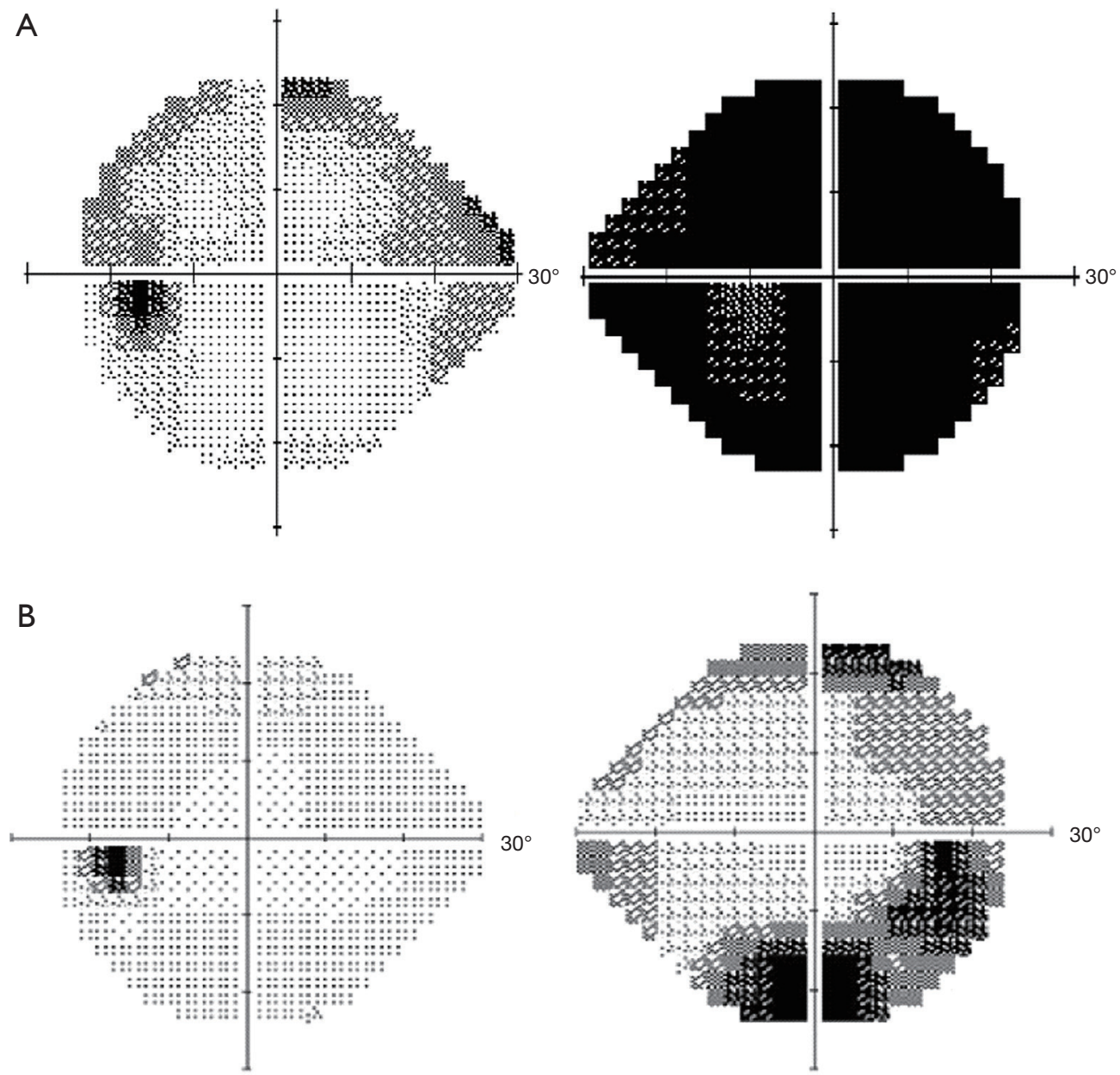

Figure 1 The 24-2 Visual Field Test showed the following before (A) and after (B) VMAT treatment. (A) Generalized visual field loss (OD) and arcuate scotoma (OS) with a MD of $-27.69 \mathrm{~dB}$ OD and $-11.73 \mathrm{~dB}$ OS; (B) residual right peripheral scotoma (OD) with $\mathrm{MD}-11.27 \mathrm{~dB}$ and a nearly normal OS with $\mathrm{MD}-0.44 \mathrm{~dB}$ were observed after treatment. Visual function in the right eye significantly increased after VMAT treatment. VMAT, volumetric modulated arc therapy; OD, oculus dexter; OS, oculus sinister; MD, mean deviation.

defined as D5\%/D95\%, in which $\mathrm{D} 5 \%$ and $\mathrm{D} 95 \%$ are the minimum doses delivered to $5 \%$ and $95 \%$ of the PTV, respectively, as previously reported (12).

A total dose of 54 Gy was delivered to the PTV in 30 fractions by the noncoplanar VMAT technique with a PCI of 0.665 and UI of 1.074. The non-coplanar plan consisted of two noncoplanar partial arcs spanned from $310-240^{\circ}$ and $240-300^{\circ}$ with couch rotations of $90^{\circ}$ and $45^{\circ}$ and collimator angles of $5^{\circ}$ and $355^{\circ}$, respectively. Compared with the other techniques, noncoplanar VMAT had the best uniformity and coverage and had fewer doses in the OAR than the other RT techniques (Table 1).

Treatment was well tolerated. From September 2017 to May 2019, no visual impairment, impaired ocular mobility, trigeminal deficit, proptosis, localized alopecia, transient worsening of headache or visual deterioration during or shortly after treatment, radiation retinopathy, cerebrovascular accident, cognitive impairment or brain necrosis were recorded. The color discrimination was improved from $1 / 15$ to $13 / 15$ after receiving $27 \mathrm{~Gy}$ in 15 fractions. The $\mathrm{VA}_{\mathrm{CC}}$ of the right eye treated with 54 Gy improved from 0.3 to 0.6 at 9 months and to 1.0 at 15 months after RT. Significant improvement of the visual field with an $\mathrm{MD}$ of $-11.27 \mathrm{~dB}$ in the right eye was noted by automated perimetry (Figure 1). The following MRI revealed a mildly altered tumor density with a stable tumor 


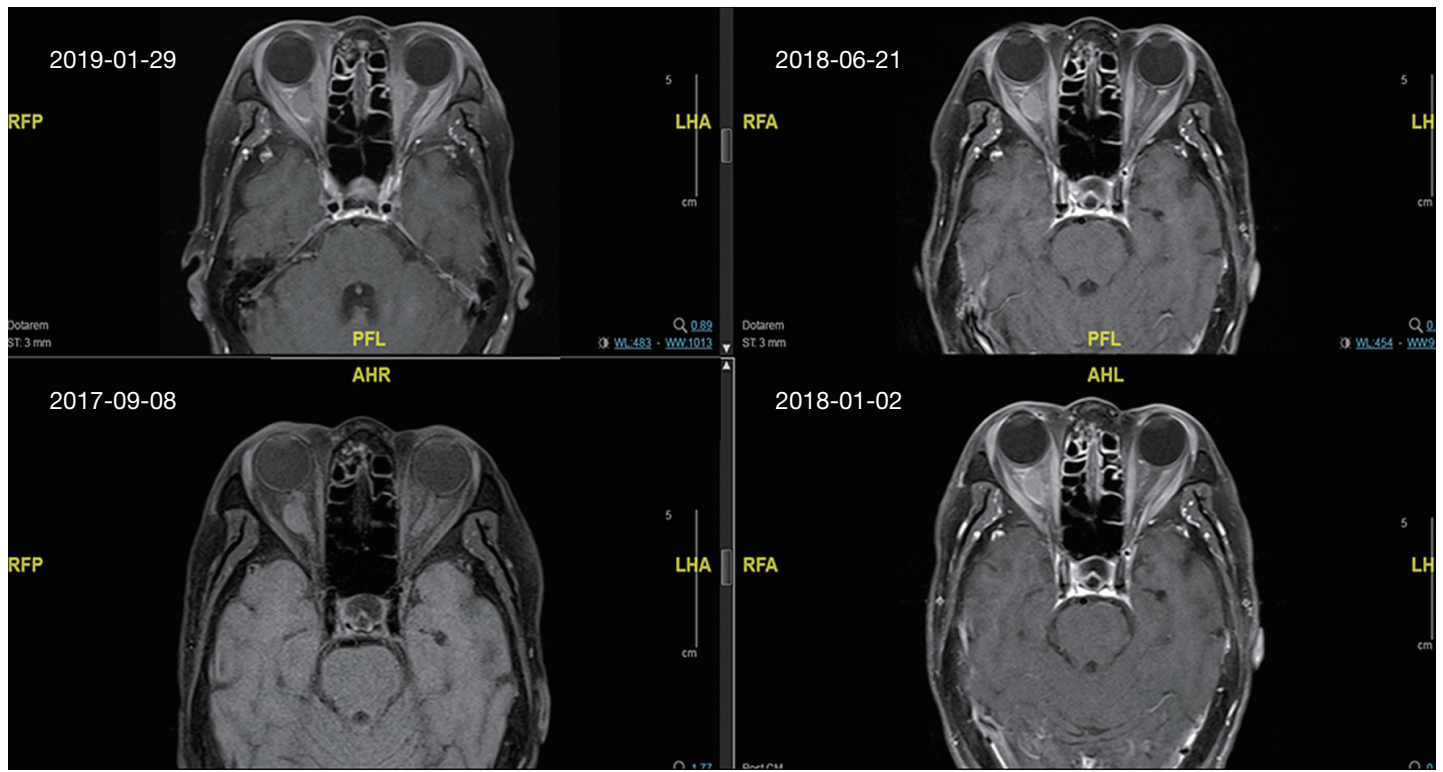

Figure 2 The MRI study performed before and after VMAT revealed a mildly altered tumor density with a stable tumor size. MRI, magnetic resonance imaging; VMAT, volumetric modulated arc therapy.
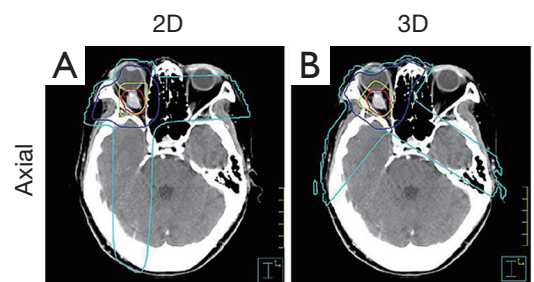

Coplanar-IMRT
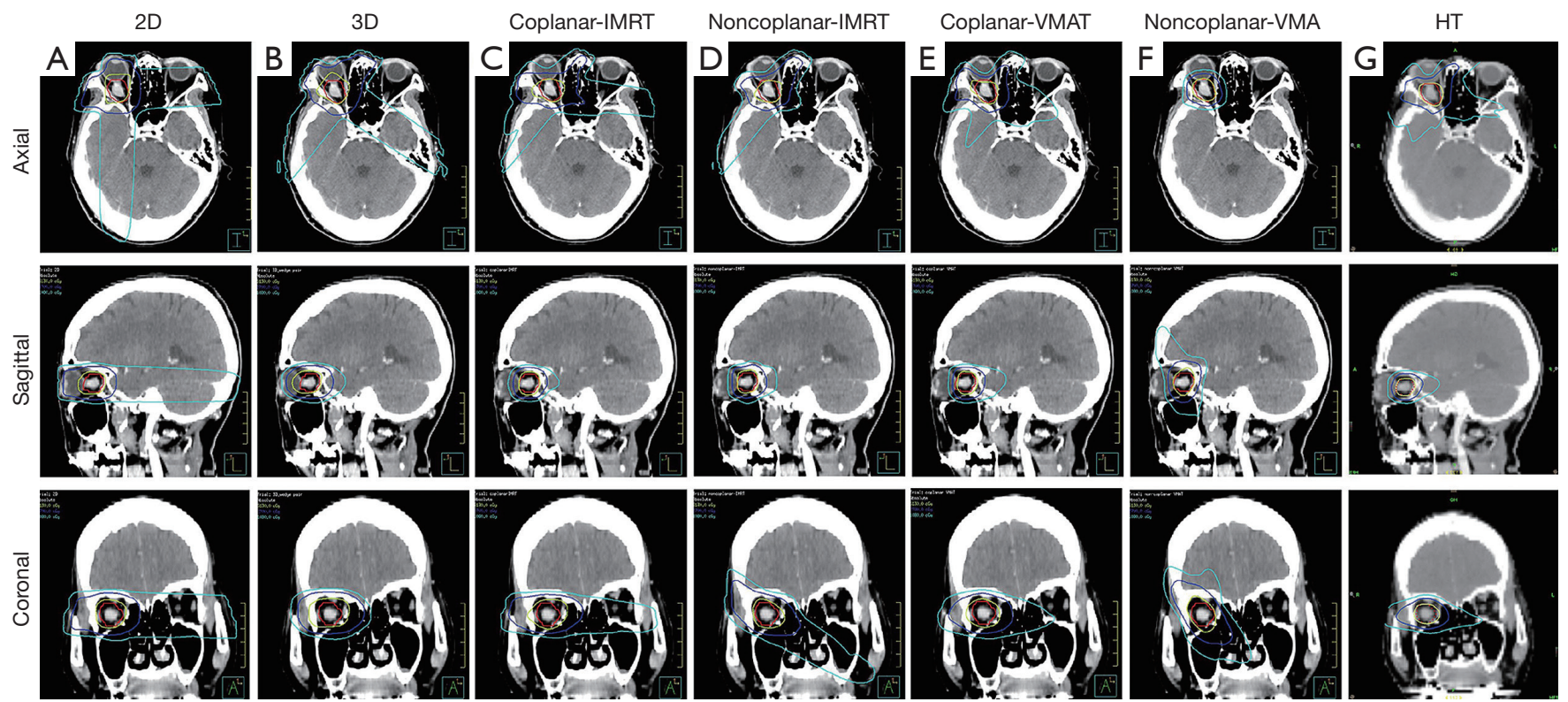

Figure 3 Isodose distributions of the prescribed dose of 54 Gy to the PTV for different treatment techniques in the transverse, sagittal and coronal views. (A) 2DRT; (B) 3DCRT; (C) coplanar IMRT; (D) noncoplanar IMRT; (E) coplanar VMAT; (F) noncoplanar VMAT; (G) helical tomotherapy. 2DRT, conventional radiation therapy; 3DCRT, three-dimensional conformal radiation therapy; IMRT, intensitymodulated radiation therapy; VMAT, volumetric modulated arc therapy.

size in the following period (Figure 2). The timeline to outline the whole process was listed in the Figure 4 to make the whole process more clear.
All procedures performed in studies involving human participants were in accordance with the ethical standards of the institutional and/or national research committee(s) 


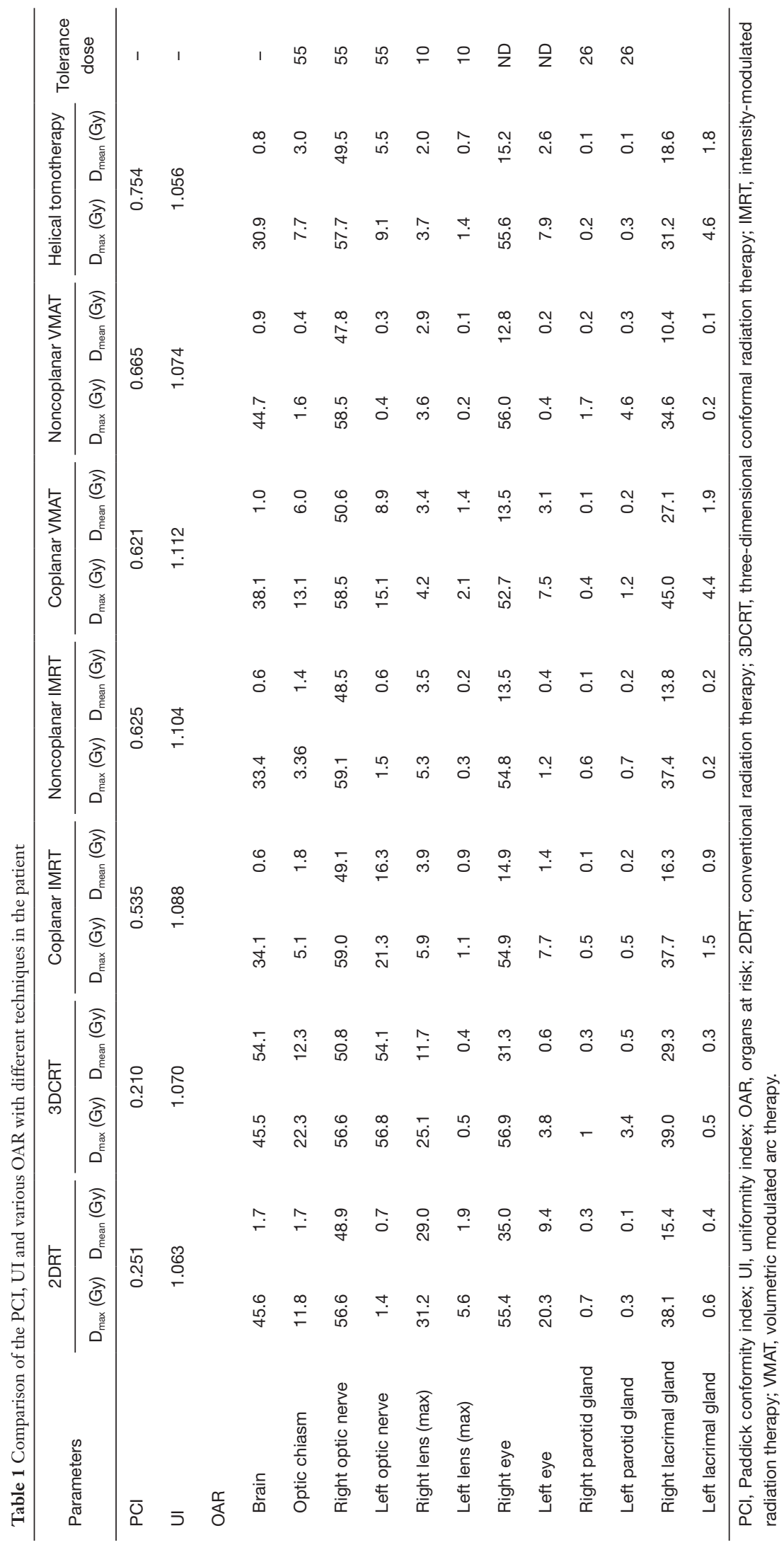




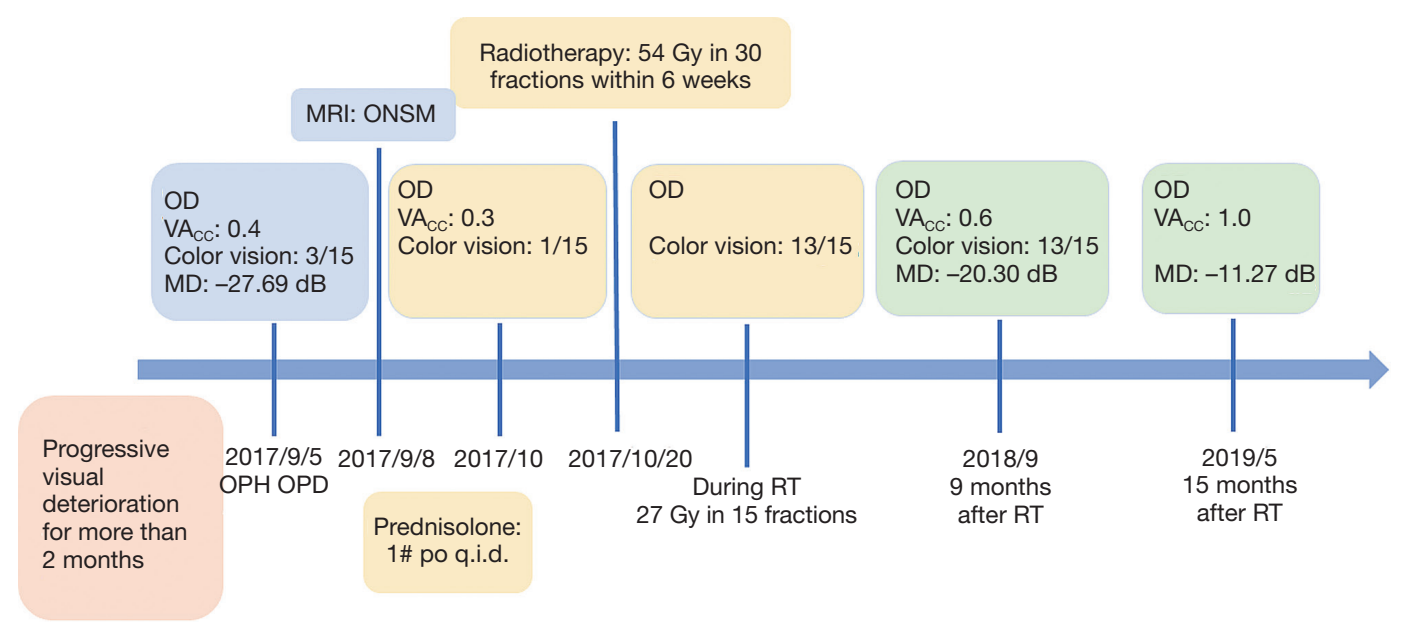

Figure 4 The timeline to outline the whole process of treatment. ONSM, optic nerve sheath meningioma; OD, oculus dexter; VA $\mathrm{CC}_{\text {, visual }}$ acuity with correction; MD, mean deviation; OPH, ophthalmology; OPD, out-patient department; RT, radiotherapy; MRI: magnetic resonance imaging.

and with the Helsinki Declaration (as revised in 2013). The need for informed consent was waived by the Institutional Review Board of the Far Eastern Memorial Hospital (FEMH-IRB-108081-C) due to the research involves no more than minimal risk to subject. The retrospective data were collected after receiving approval from the Institutional Review Board of the Far Eastern Memorial Hospital.

\section{Discussion}

This is the first report of a patient with ONSM treated by a noncoplanar VMAT technique with an encouraging response. The treatment was noninvasive and well tolerated. A benefit of vision recovery to normal was observed at 15 months after the VMAT technique. Additionally, no severe complications were noted during or after RT in the following period.

The annual respective incidence of meningioma for males and females reported by the Central Brain Tumor Registry of the United States (CBTRUS) is 1.8 and 3.4 per 100,000 people, respectively, and most cases are often discovered in middle-to late adult life (13). ONSMs can be categorized among grade I meningiomas using the WHO grading scale (14). The first effective results using RT to treat ONSM were reported by Smith et al. (15). In studies with case numbers larger than 20 patients, the efficacy with visual function and a visual field that remained stable or improved was $92-100 \%(8,9,16)$. Additionally, the radiologically stable disease rate was $92-95 \%$ and the rate of acute or late treatment-related toxicity was $2-33 \%(9,16)$. However, these data mentioned above mostly resulted from treatment with 3DCRT or FSRT. Recently, patients with ONSM were treated by IMRT $(7,17)$ or image-guided RT (18) with promising results. IMRT improves the dose distributions achieved using 3DCRT. Nevertheless, IMRT treatment techniques (step-and-shoot and sliding window) are fixed-gantry techniques. Recently, the University of Texas MD Anderson Cancer Center compared the plans between proton therapy and coplanar VMAT for ONSM treatment and favored VMAT after considering the conformity and uniformity (19). In a previous report, the noncoplanar IMRT technique reduced the off-target dose to normal organs at a statistically significant level compared to IMRT and helical tomotherapy (20).

In our case, treatment planning with different techniques including 2DRT, 3DCRT, coplanar IMRT, noncoplanar IMRT, coplanar VMAT, noncoplanar VMAT, and helical tomotherapy were compared (Table 1). PCI of noncoplanar IMRT, coplanar VMAT, noncoplanar VMAT, and helical tomotherapy were above 0.6 which was better than PCI of 2DRT, 3DCRT, and coplanar IMRT. However, UI of noncoplanar IMRT and coplanar VMAT were higher than 1.1 which was higher than other techniques. Considering normal tissue sparing, noncoplanar VMAT provided significant better protection of vision associated organ and structure including optic chiasm, left optic nerve, bilateral lens, and left eye than other treatment techniques. 
Moreover, in the evaluation for isodose distribution, noncoplanar-VMAT provided the best conformal isodose line than other techniques in axial image (Figure 3). Comparing with helical tomotherapy, isodose distribution of noncoplanar-VMAT tends to be more perpendicular to optic nerve pathway and leading less dose deposits to optic nerve track. Helical tomotherapy, however, causing dose spreading more parallel along with optic nerve track leading to higher dose effect on vision associated organs. After evaluated the balances between optimal tumor coverage and critical organs sparing with minimal off-target irradiation, noncoplanar VMAT plan was decided.

Treatment was well tolerated without comorbidity. Additionally, the color discrimination and $\mathrm{VA}_{\mathrm{CC}}$ of the right eye were recovered as normal. Although the study of Al Feghali et al. (19), along with our current observation, suggests that the VMAT technique can efficiently treat ONSM, the number of patients is limited, and a longer follow-up is needed.

Here, we describe the first successful treatment of an ONSM using a noncoplanar VMAT technique, which appears to be promising for the management of such tumors. The tolerance and safety were excellent, and further investigation is warranted to assess potential late effects.

\section{Acknowledgments}

Funding: This study was supported by research grants from Far Eastern Memorial Hospital grants (FEMH108-2314-B-418-003-MY2). The role of the funding was in the collection and analysis of data and in writing the manuscript.

\section{Footnote}

Provenance and Peer Review: This article was commissioned by the editorial office, Therapeutic Radiology and Oncology for the series "Precision Medicine for Cancer". The article has undergone external peer review.

Reporting Checklist: The authors have completed the CARE reporting checklist. Available at http://dx.doi.org/10.21037/ tro-2019-pmc-07

Conflicts of Interest: All authors have completed the ICMJE uniform disclosure form (available at http://dx.doi. org/10.21037/tro-2019-pmc-07). The series "Precision Medicine for Cancer" was commissioned by the editorial office without any funding or sponsorship. $\mathrm{CHH}$ served as the unpaid Guest Editor of the series and serves as an unpaid editorial board member of Therapeutic Radiology and Oncology from May 2020 to Apr 2022. HHN served as the unpaid Guest Editor of the series. The authors have no other conflicts of interest to declare.

Etbical Statement: The authors are accountable for all aspects of the work in ensuring that questions related to the accuracy or integrity of any part of the work are appropriately investigated and resolved. All procedures performed in studies involving human participants were in accordance with the ethical standards of the institutional and/or national research committee(s) and with the Helsinki Declaration (as revised in 2013). The study was approved by the Institutional Review Board of our hospital (FEMH108081-C). The need for informed consent was waived by the Institutional Review Board of the Far Eastern Memorial Hospital, and retrospective data were collected after receiving approval from the Institutional Review Board of the Far Eastern Memorial Hospital.

Open Access Statement: This is an Open Access article distributed in accordance with the Creative Commons Attribution-NonCommercial-NoDerivs 4.0 International License (CC BY-NC-ND 4.0), which permits the noncommercial replication and distribution of the article with the strict proviso that no changes or edits are made and the original work is properly cited (including links to both the formal publication through the relevant DOI and the license). See: https://creativecommons.org/licenses/by-nc-nd/4.0/.

\section{References}

1. Dutton JJ. Optic nerve sheath meningiomas. Surv Ophthalmol 1992;37:167-83.

2. Saeed P, Rootman J, Nugent RA, et al. Optic nerve sheath meningiomas. Ophthalmology 2003;110:2019-30.

3. Jeremic B, Pitz S. Primary optic nerve sheath meningioma: stereotactic fractionated radiation therapy as an emerging treatment of choice. Cancer 2007;110:714-22.

4. Kennerdell JS, Maroon JC, Malton M, et al. The management of optic nerve sheath meningiomas. Am J Ophthalmol 1988;106:450-7.

5. Cristante L. Surgical treatment of meningiomas of the orbit and optic canal: a retrospective study with particular attention to the visual outcome. Acta Neurochir (Wien) 1994;126:27-32. 
6. Maguire PD, Clough R, Friedman AH, et al. Fractionated external-beam radiation therapy for meningiomas of the cavernous sinus. Int J Radiat Oncol Biol Phys 1999;44:75-9.

7. Jin J, Joo JD, Han JH, et al. Optic nerve sheath meningioma: preliminary analysis of the role of radiation therapy. Brain Tumor Res Treat 2018;6:8-12.

8. Soldà F, Wharram B, De Ieso PB, et al. Long-term efficacy of fractionated radiotherapy for benign meningiomas. Radiother Oncol 2013;109:330-4.

9. Bloch O, Sun M, Kaur G, et al. Fractionated radiotherapy for optic nerve sheath meningiomas. J Clin Neurosci 2012;19:1210-5.

10. Elith C, Dempsey SE, Findlay N, et al. An introduction to the intensity-modulated radiation therapy (IMRT) techniques, tomotherapy, and VMAT. J Med Imaging Radiat Sci 2011;42:37-43.

11. Paddick I. A simple scoring ratio to index the conformity of radiosurgical treatment plans. Technical note. J Neurosurg 2000;93 Suppl 3:219-22.

12. Wang X, Zhang X, Dong L, et al. Effectiveness of noncoplanar IMRT planning using a parallelized multiresolution beam angle optimization method for paranasal sinus carcinoma. Int J Radiat Oncol Biol Phys 2005;63:594-601.

13. Surawicz TS, McCarthy BJ, Kupelian V, et al. Descriptive epidemiology of primary brain and CNS tumors: results from the Central Brain Tumor Registry of the United

doi: 10.21037/tro-2019-pmc-07

Cite this article as: Wu CY, Huang TL, Nien HH, Shueng PW, Tien HJ, Yeh HP, Hsieh CH. Noncoplanar volumetric modulated arc therapy for patient with optic nerve sheath meningioma: a case report and literature review. Ther Radiol Oncol 2020;4:18.
States, 1990-1994. Neuro Oncol 1999;1:14-25.

14. Louis DN, Perry A, Reifenberger G, et al. The 2016 World Health Organization Classification of Tumors of the Central Nervous System: a summary. Acta Neuropathol 2016;131:803-20.

15. Smith JL, Vuksanovic MM, Yates BM, et al. Radiation therapy for primary optic nerve meningiomas. J Clin Neuroophthalmol 1981;1:85-99.

16. Arvold ND, Lessell S, Bussiere M, et al. Visual outcome and tumor control after conformal radiotherapy for patients with optic nerve sheath meningioma. Int J Radiat Oncol Biol Phys 2009;75:1166-72.

17. Inoue T, Mimura O, Masai N, et al. Early intervention using high-precision radiotherapy preserved visual function for five consecutive patients with optic nerve sheath meningioma. Int J Clin Oncol 2018;23:826-34.

18. Lo AT, Gibbs IC, Soltys SG, et al. IGRT for optic nerve sheath meningioma. Int J Radiat Oncol Biol Phys 2012;84:S763.

19. Al Feghali KA, Yeboa DN, Chasen B, et al. The use of $68 \mathrm{Ga}$-DOTATATE PET/CT in the non-invasive diagnosis of optic nerve sheath meningioma: a case report. Front Oncol 2018;8:454.

20. Hsieh CH, Liu CY, Shueng PW, et al. Comparison of coplanar and noncoplanar intensity-modulated radiation therapy and helical tomotherapy for hepatocellular carcinoma. Radiat Oncol 2010;5:40. 